\title{
Capabilities of multi-pinhole SPECT with two stationary detectors for in vivo rat imaging
}

\author{
Jan P. Janssen ${ }^{1,2,7}$, Jan V. Hoffmann $n^{1,2,7}$, Takayuki Kanno 2,3 , Naoko Nose ${ }^{4}$, Jan-Peter Grunz ${ }^{5}$, \\ Masahisa Onoguchi ${ }^{3}$, Xinyu Chen ${ }^{2,6}$, Constantin Lapa ${ }^{6}$, Andreas K. Buck ${ }^{1} \&$ \\ Takahiro Higuchi ${ }^{1,2,4 凶}$
}

We aimed to investigate the image quality of the U-SPECT5/CT E-Class a micro single-photon emission computed tomography (SPECT) system with two large stationary detectors for visualization of rat hearts and bones using clinically available ${ }^{99 \mathrm{~m} T c-l a b e l l e d ~ t r a c e r s . ~ S e n s i t i v i t y, ~ s p a t i a l ~ r e s o l u t i o n, ~}$ uniformity and contrast-to-noise ratio (CNR) of the small-animal SPECT scanner were investigated in phantom studies using an ultra-high-resolution rat and mouse multi-pinhole collimator (UHR-RM). Point source, hot-rod, and uniform phantoms with ${ }^{99 \mathrm{~m}} \mathrm{Tc}$-solution were scanned for high-count performance assessment and count levels equal to animal scans, respectively. Reconstruction was performed using the similarity-regulated ordered-subsets expectation maximization (SROSEM) algorithm with Gaussian smoothing. Rats were injected with $\sim 100 \mathrm{MBq}\left[{ }^{99 \mathrm{~m}} \mathrm{Tc}\right] \mathrm{Tc}-\mathrm{MIBI}$ or $\sim 150 \mathrm{MBq}$ $\left.{ }^{[99 \mathrm{~m}} \mathrm{Tc}\right] \mathrm{TC}-\mathrm{HMDP}$ and received multi-frame micro-SPECT imaging after tracer distribution. Animal scans were reconstructed for three different acquisition times and post-processed with different sized Gaussian filters. Following reconstruction, CNR was calculated and image quality evaluated by three independent readers on a five-point scale from $1=$ "very poor" to $5=$ "very good". Point source sensitivity was $567 \mathrm{cps} / \mathrm{MBq}$ and radioactive rods as small as $1.2 \mathrm{~mm}$ were resolved with the UHR-RM collimator. Collimator-dependent uniformity was 55.5\%. Phantom CNR improved with increasing rod size, filter size and activity concentration. Left ventricle and bone structures were successfully visualized in rat experiments. Image quality was strongly affected by the extent of post-filtering, whereas scan time did not have substantial influence on visual assessment. Good image quality was achieved for resolution range greater than $1.8 \mathrm{~mm}$ in bone and $2.8 \mathrm{~mm}$ in heart. The recently introduced small animal SPECT system with two stationary detectors and UHR-RM collimator is capable to provide excellent image quality in heart and bone scans in a rat using standardized reconstruction parameters and appropriate post-filtering. However, there are still challenges in achieving maximum system resolution in the sub-millimeter range with in vivo settings under limited injection dose and acquisition time.

Preclinical single-photon emission computed tomography (SPECT) imaging is an evolving field full of challenges. The introduction of pinhole collimation opened up new applications for small-animal SPECT imaging ${ }^{1-3}$ and with the development of multi-pinhole collimation, high spatial resolution in the sub-millimeter range with acceptable sensitivity was possible ${ }^{3-6}$. Combining sub-millimeter precision imaging with a plethora of easily accessible radioisotopes and the option of detecting multiple radioisotopes simultaneously set SPECT apart from its competitor positron emission tomography $(\mathrm{PET})^{7}$. Further, the use of stationary detectors covering $360^{\circ}$ of a fixed field of view (FOV) enables dynamic studies with increased precision ${ }^{8,9}$, while reducing mechanical issues and complex system maintenance compared to a setup with moving parts ${ }^{10}$. The previous

\footnotetext{
${ }^{1}$ Department of Nuclear Medicine, University Hospital Würzburg, Oberdürrbacher Strasse 6, 97080 Würzburg, Germany. ${ }^{2}$ Comprehensive Heart Failure Centre, University Hospital Würzburg, Würzburg, Germany. ${ }^{3}$ Department of Quantum Medical Technology, Graduate School of Medical Sciences, Kanazawa University, Kanazawa, Japan. ${ }^{4}$ Graduate School of Medicine, Dentistry and Pharmaceutical Sciences, Okayama University, Okayama, Japan. ${ }^{5}$ Department of Diagnostic and Interventional Radiology, University Hospital Würzburg, Würzburg, Germany. ${ }^{6}$ Nuclear Medicine, Medical Faculty, University of Augsburg, Augsburg, Germany. ${ }^{7}$ These authors contributed equally: Jan P. Janssen and Jan V. Hoffmann. ${ }^{\circledR}$ email: higuchi_t@ukw.de
} 


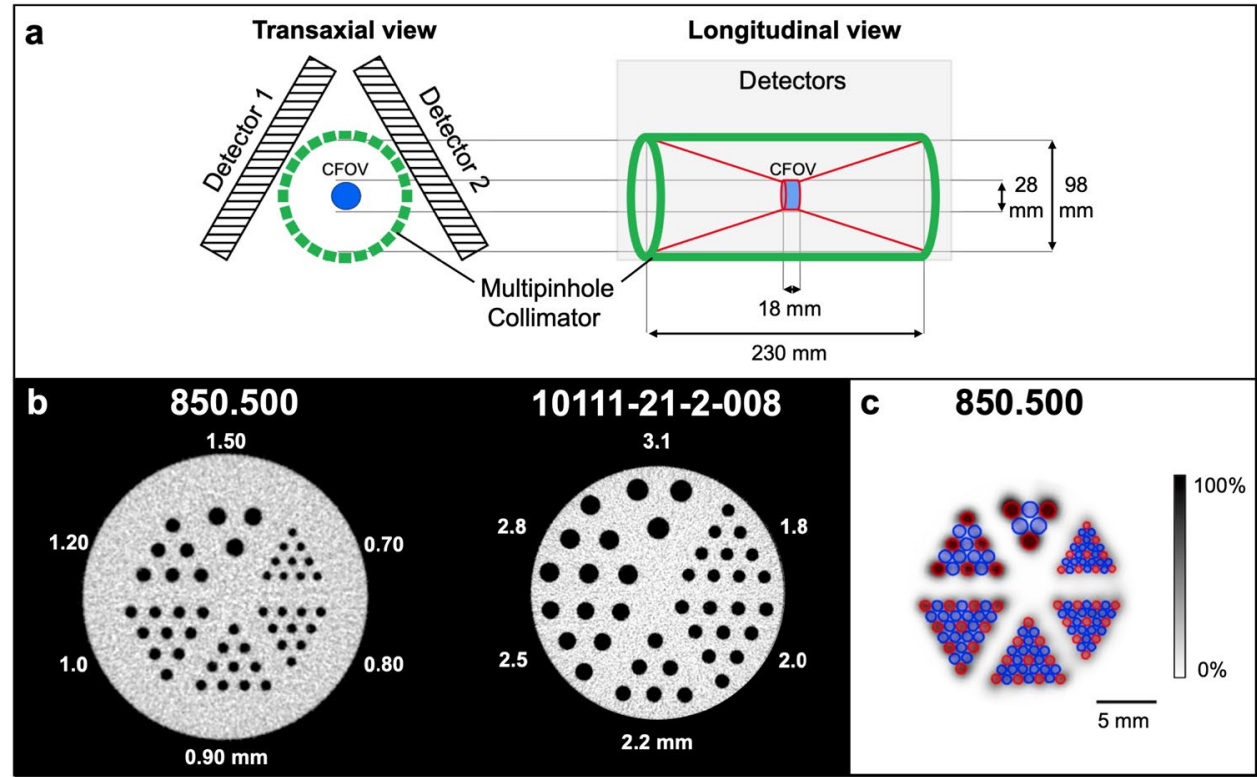

Figure 1. Scanner construction, mini Derenzo phantoms and contrast-to-noise ratio method. (a) Illustration of the arrangement of the U-SPECT5-E with two stationary detectors in two axes. Each detector, illustrated here as a square, has a surface of $472 \mathrm{~mm} \times 595 \mathrm{~mm}$ and contains $\mathrm{NaI}(\mathrm{Tl})$ crystals with a thickness of $9.5 \mathrm{~mm}$. The bore diameter of the UHR-RM collimator is $98 \mathrm{~mm}$. Center field-of-view (CFOV) measures $28 \mathrm{~mm}$ in diameter and $18 \mathrm{~mm}$ in length. Scan volume can be manually adjusted to a maximum length of $230 \mathrm{~mm}$. (b) Transaxial view of CT images of the mini Derenzo phantoms 850.500 and 10111-21-2-008 with their respective rod sizes. The shown phantoms were used to determine the maximum resolution and contrast-to-noise ratio (CNR) for various count levels. Based on CT images, the templates for CNR analysis were created. (c) SPECT image of the phantom 850.500 with the template for CNR analysis consisting of radioactive (red) and non-radioactive (blue) regions of interest (ROIs). ROIs have 0.9 times the diameter of corresponding rod sizes, length is $6.0 \mathrm{~mm}$. This example SPECT image has a slice thickness of $6.0 \mathrm{~mm}$ and was taken with $285.22 \mathrm{MBq} / \mathrm{mL}, 300 \mathrm{~s}$ time per bed position and 9 bed positions.

generations of these ultra-high-resolution SPECT systems usually have three large stationary detectors in a triangular configuration ${ }^{10,11}$. For this work, however, we assessed the image quality of a cost-efficient scanner that omits the bottom detector.

High spatial resolution can improve the diagnostic value of SPECT scans if sufficient image quality is achieved, which is particularly affected by the choice of collimator ${ }^{12}$, reconstruction algorithm ${ }^{5,13}$, post-reconstruction filter $^{13}$ and injection dose ${ }^{14}$. Hence, we evaluated the influence of acquisition time and Gaussian post-filtering, using a novel iterative reconstruction algorithm with similarity-regulated ordered-subsets expectation maximization (SROSEM), that enables constant reconstruction parameters for a wide range of activity concentrations ${ }^{15}$.

Basic and translational researchers have been mainly using small-animal models such as mice or rats to reduce housing and maintenance costs, to explore pathophysiology and to develop new drugs. Rats have certain potential advantages over mice ${ }^{16}$. It is easier to develop suitable invasive devices for surgical procedures and hemodynamic measurements and offers larger tissue mass for histological and biological analyses. Furthermore, recent transfer of functional genomics technology into rats reemphasizes the potential of rat models ${ }^{16}$. For animal SPECT imaging, although bigger size of the organs is an advantage of rats over mice, the higher soft tissue attenuation and scattering, as well as requirement of larger bore and transaxial size, might have negative impact on precise imaging.

This study aims to examine the preclinical applicability and image quality of the recently introduced microSPECT system for rat imaging under in vivo conditions using $\left[{ }^{99 \mathrm{~m}} \mathrm{Tc}\right] \mathrm{Tc}-\mathrm{MIBI}^{17}$ and $\left[{ }^{99 \mathrm{~m}} \mathrm{Tc}\right] \mathrm{Tc}-\mathrm{HMDP}{ }^{18,19}$ with a pre-set SROSEM reconstruction algorithm and Gaussian post-filtering.

\section{Materials and methods}

System description. U-SPECT5/CT E-Class (referred to as "U-SPECT5-E"; MILabs, Utrecht, The Netherlands) is an ultra-high-resolution SPECT system for preclinical imaging of small- to medium-sized animals. While the scanner architecture is based on previous generations of micro-SPECT systems (U-SPECT-II; $\mathrm{U}-S P E C T^{+}$; MILabs) ${ }^{10,11}$, the conventional U-SPECT5 system features three stationary detectors with a size of $472 \mathrm{~mm} \times 595 \mathrm{~mm}$ and $9.5 \mathrm{~mm}$ thick thallium-doped sodium iodide $(\mathrm{NaI}(\mathrm{Tl}))$ scintillation crystals arranged in a triangular format around the FOV. Striving for cost effectiveness, the U-SPECT5-E is built without the bottom detector as illustrated in Fig. 1a.

A centrally located collimator with multi-pinhole configuration allows the acquisition of SPECT images in a spiral step mode using the XYZ stage ${ }^{20}$. 
In this study, an ultra-high resolution rat/mouse (UHR-RM) collimator made of tungsten was used with 75 pinholes (diameter of $1.0 \mathrm{~mm}$ ) in 5 rows, all pointing towards a center FOV of $28 \mathrm{~mm}$ diameter and $18 \mathrm{~mm}$ length. Due to the missing bottom detector only the upper 50 pinholes contribute to the imaging process.

Data acquisition and processing. All images were acquired in list-mode and reconstructed with the SROSEM algorithm. For simplifying the reconstruction process, we used four iterations with 128 subsets and a voxel size of $0.4 \mathrm{~mm}^{3}$, subsequently applying Gaussian post-filtering as advised by Vaissier et al. ${ }^{15}$. This approach showed no perceptible decrease in image quality compared to the reference standard of maximum likelihood expectation maximization (MLEM) ${ }^{21}$.

For scatter correction, we applied the triple energy window method ${ }^{22}$ with a photopeak window of $126 \mathrm{keV}$ to $154 \mathrm{keV}$, a lower background window of $120.4 \mathrm{keV}$ to $126 \mathrm{keV}$ and an upper one of $154 \mathrm{keV}$ to $159.6 \mathrm{keV}$. The obtained SPECT images were transferred to a workstation for further Gaussian post-filtering and analysis using AMIDE (version 1.0.5 for MacOS; open source) ${ }^{23}$.

Performance measurements. Sensitivity was examined by using a point source with a ${ }^{99 m} \mathrm{Tc}$-solution of $2.7 \mathrm{MBq}$. Scan time was $5 \mathrm{~min}$ with 1 bed position (BP). Calculation was based on the National Electrical Manufacturers Association (NEMA) ${ }^{24}$ with $R_{i}$ representing the detected photopeak countrate and $A_{\text {cal }}$ standing for the total activity of the point source determined by a dose calibrator (ISOMED 2010, NUVIA Instruments, Dresden, Germany).

$$
\text { Sensitivity }=\frac{R_{i}}{A_{c a l}}
$$

Figure $1 \mathrm{~b}$ shows the mini Derenzo hot-rod phantom (850.500) that was used to investigate the spatial resolution. It was filled with a ${ }^{99 \mathrm{~m}} \mathrm{Tc}$-solution with an activity concentration of $285.2 \mathrm{MBq} / \mathrm{mL}$. Scan time was $45 \mathrm{~min}$ with 9 BP. In the visual analysis of the reconstructed data, maximum resolution was assessed as the smallest distinguishable rod size.

Uniformity was investigated by using the cylindrical container of the mini Derenzo phantom. The filling volume was $10.6 \mathrm{~mL}$ with a ${ }^{99 \mathrm{~m} T c}$-solution of $315.0 \mathrm{MBq}$ and the scan time was 45 min with 9 BP. Gaussian post-filtering was applied with a full width at half maximum (FWHM) of $1.2 \mathrm{~mm}$ representing the maximum resolution. For calculation, a cylindrical region of interest (ROI) was placed centrally in the phantom, measuring $18 \mathrm{~mm}$ in diameter and $10 \mathrm{~mm}$ in length. Collimator-dependent system uniformity was calculated as recommended by $\mathrm{NEMA}^{24}$.

$$
\text { Uniformity }(\%)=100 \times \frac{\text { Max count }- \text { Min count }}{\text { Max count }+ \text { Min count }}
$$

Phantom image quality. To evaluate the in vitro image quality, a contrast-to-noise ratio (CNR) analysis was carried out by using two mini Derenzo phantoms. This technique, as shown in Fig. 1c, was described initially by Walker et al. ${ }^{25}$. Using a high-resolution computed tomography (CT) image as a template, ROIs of 6 mm length were placed in the center of the rods. All ROIs have a diameter of 0.9 times the size of the respective radioactive rod. In addition, ROIs of the same size were placed in the non-radioactive regions in-between two radioactive rods.

The contrast $C_{d}$ was defined as:

$$
C_{d}=\frac{\overline{R_{d}}-\overline{B_{d}}}{\overline{R_{d}}}
$$

$\bar{R}_{d}$ is the mean value of all radioactive ROIs and $\overline{B_{d}}$ is the mean value of all non-radioactive ROIs for the rod size $d$. The noise $N_{d}$ was defined as:

$$
N_{d}=\frac{\sqrt{\sigma_{R_{d}}^{2}+\sigma_{B_{d}}^{2}}}{\overline{R O I s_{d}}}
$$

$\sigma_{R_{d}}$ and $\sigma_{B_{d}}$ are the standard deviations in radioactive and non-radioactive ROIs, while $R \overline{O I} s_{d}$ is the mean value of all ROIs of a rod size $d$, no matter if they are radioactive or non-radioactive.

These two formulas are used to calculate the CNR:

$$
C N R_{d}=\frac{C_{d}}{N_{d}}
$$

This image quality analysis was performed for the smaller 850.500 and the larger 10111-21-2-008 phantom with a rod size range of $0.7-1.5 \mathrm{~mm}$ and $1.8-3.1 \mathrm{~mm}$, respectively. In addition to high-count performance measurements with $\sim 290.0 \mathrm{MBq} / \mathrm{mL}$, low-count measurements were also conducted $(\sim 1.0 \mathrm{MBq} / \mathrm{mL}, \sim 0.5 \mathrm{MBq} /$ $\mathrm{mL}, \sim 0.1 \mathrm{MBq} / \mathrm{mL}$ ). To minimize the loss of resolution, images were optimized by a Gaussian post-filter for each rod size. The kernel size of the filter always corresponded to FWHM = rod size. 
a

\section{transverse}

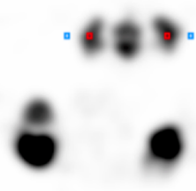

b

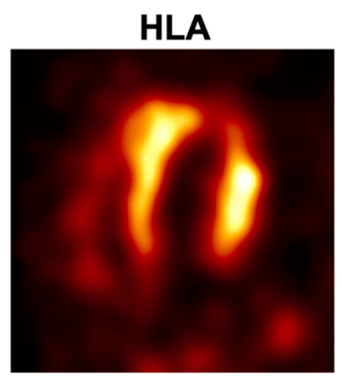

coronal

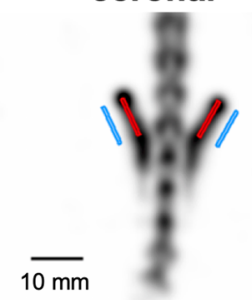

SA

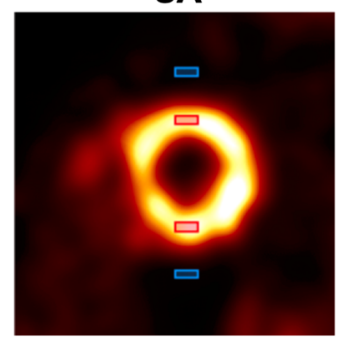

sagittal
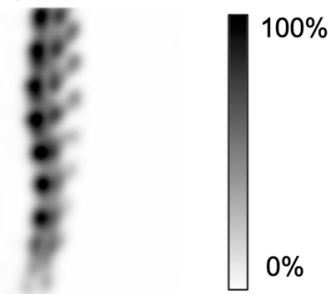

$0 \%$

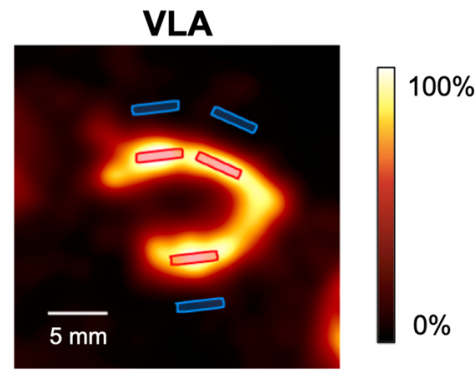

Figure 2. In vivo contrast-to-noise ratio method. (a) [ $\left.{ }^{99 \mathrm{~m}} \mathrm{Tc}\right] \mathrm{Tc}-\mathrm{HMDP}$ SPECT image of the lower spine and pelvis region of a healthy rat from transverse, coronal and sagittal perspective. Illustration of the in vivo contrast-to-noise ratio (CNR) analysis for the focused bone scan by using radioactive (red) and non-radioactive (blue) regions of interest (ROIs) with a size of $0.8 \times 8.0 \times 1.2 \mathrm{~mm}^{3}$ each. The rat was injected with $154.9 \mathrm{MBq}$ [ $\left.{ }^{99 \mathrm{~m}} \mathrm{Tc}\right] \mathrm{Tc}-\mathrm{HMDP}$ and scanned $60 \mathrm{~min}$ post injection for $90 \mathrm{~min}$ total scan time. (b) [ $\left.{ }^{99 \mathrm{~m}} \mathrm{Tc}\right] \mathrm{Tc}-\mathrm{MIBI}$ SPECT image of a healthy rat cropped and rotated to the horizontal long axis (HLA), short axis (SA) and vertical long axis (VLA) view of the heart. The ROIs for CNR analysis in the heart had a size of $4.0 \times 0.8 \times 2.0 \mathrm{~mm}^{3}$ each. The rat was injected with $108.5 \mathrm{MBq}\left[{ }^{99 \mathrm{~m}} \mathrm{Tc}\right] \mathrm{Tc}-\mathrm{MIBI}$ and scanned $25 \mathrm{~min}$ post injection for $60 \mathrm{~min}$ total scan time. Reconstructed images were filtered with a Gaussian filter (full width at half maximum (FWHM) $=2.2 \mathrm{~mm}$ ) and are shown with a slice thickness of $0.4 \mathrm{~mm}$.

Animal studies. Animal protocols were approved by the local Animal Care and Use Committee (Regierung von Unterfranken, Germany) and conducted according to the Guide for the Care and Use of Laboratory Animals ${ }^{26}$.

[99m Tc]Tc-HMDP bone rat imaging. One healthy female Wistar rat (Charles River Laboratories, Sulzfeld, Germany) with $217.0 \mathrm{~g}$ body weight was injected $154.86 \mathrm{MBq}$ of $\left[{ }^{99 \mathrm{~m}} \mathrm{Tc}\right.$ ] Tc-HMDP ${ }^{18,19}$ via tail vein. Acquisition started at $1 \mathrm{~h}$ post-injection with a total scan time of $90 \mathrm{~min}$ containing 185 -min-frames with $20 \mathrm{~s}$ time per bed position (TPB) and 15 BP. During acquisition, the rat underwent an inhalation anesthesia (2.0\% isoflurane, 1.5 $\mathrm{L} \mathrm{O}_{2} / \mathrm{min}$ ).

To determine the CNR in the reconstructed bone images, one radioactive ROI was placed centrally in the pelvic bone on each side and a corresponding non-radioactive ROI was placed in the background at $4 \mathrm{~mm}$ distance. All ROIs in the bone scan were box-shaped and had a size of $0.8 \times 8.0 \times 1.2 \mathrm{~mm}^{3}$ (Fig. $2 \mathrm{a}$ ). This analysis was applied to the focused bone scan with reconstructed frame lengths of 5,30 and $90 \mathrm{~min}$. The three reconstructions were analyzed unfiltered and for five different Gaussian post-filters (1.2, 1.8, 2.2, 2.8 and $3.5 \mathrm{~mm})$.

[99m Tc]Tc-MIBI cardiac rat imaging. Another healthy female Wistar rat (Charles River Laboratories), weighing $231.5 \mathrm{~g}$, was injected $108.51 \mathrm{MBq}$ of $\left[{ }^{99 \mathrm{~m}} \mathrm{Tc}\right] \mathrm{Tc}-\mathrm{MIBI}^{17}$ into the tail vein. Twenty-five minutes after injection, a total-body scan was performed with an acquisition time of $60 \mathrm{~min}$, divided into six 10-min-frames (TPB $15 \mathrm{~s}, \mathrm{BP} 40)$. While scanning, an inhalation anesthesia (2.0\% isoflurane, $\left.1.5 \mathrm{~L} \mathrm{O}_{2} / \mathrm{min}\right)$ was carried out. By using the heart uptake of [ $\left.{ }^{99 \mathrm{~m}} \mathrm{Tc}\right] \mathrm{Tc}-\mathrm{MIBI}$ described in the literature with $1.71 \pm 0.63 \% \mathrm{ID} / \mathrm{g}$ for the myocardium of the $\mathrm{rat}^{27}$, we calculated the expected activity in the rat heart to match it with the phantom studies, taking into account the different TPB.

For the reconstructed data, three radioactive ROIs were placed centrally in the wall of the left ventricle and three non-radioactive ROIs were each positioned $4 \mathrm{~mm}$ away from the corresponding radioactive ROI in the background outside of the heart. All ROIs in the heart scan were box-shaped and had a size of $4.0 \times 0.8 \times 2.0 \mathrm{~mm}^{3}$ (Fig. 2b). This analysis was applied to the whole-body heart scan with frame lengths of 10, 30 and 60 min. Again, all three images were analyzed unfiltered and for five different Gaussian post-filters (1.2, 2.2, 2.8, 3.5 and $4.0 \mathrm{~mm})$. 
a

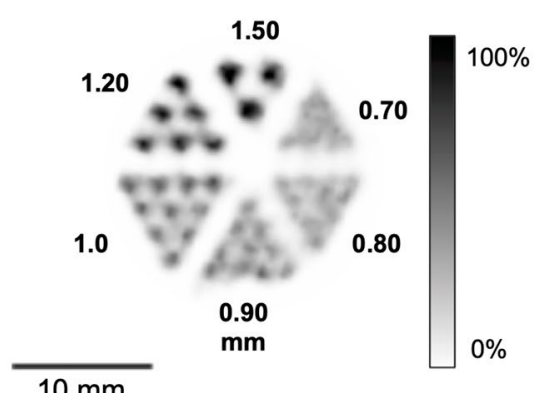

$10 \mathrm{~mm}$ b

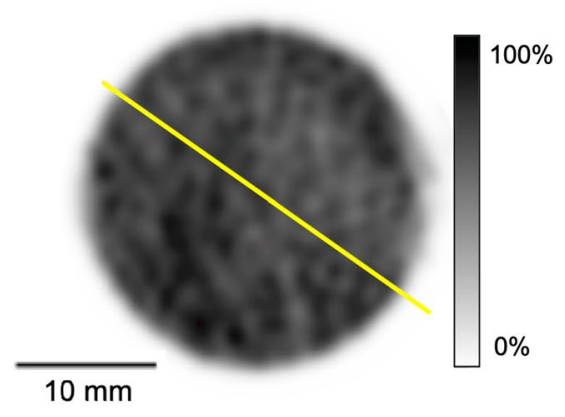

C

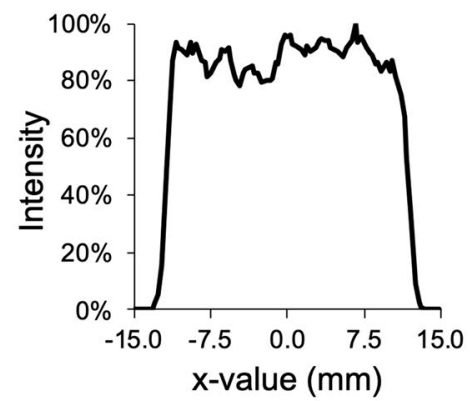

Figure 3. Resolution and uniformity. (a) shows a representative SPECT image of the hot-rod phantom (850.500), filled with $285.2 \mathrm{MBq} / \mathrm{mL}^{99 \mathrm{~m}} \mathrm{Tc}$-solution to analyze maximum resolution. Minimal discriminable rod size was $1.2 \mathrm{~mm}$. Uniformity phantom (b), filled homogenously with $29.7 \mathrm{MBq} / \mathrm{mL}^{99 \mathrm{~m} T c}$-solution and line profile (c) corresponding to the yellow line. Gaussian post-filter equals maximum resolution of $1.2 \mathrm{~mm}$.

In vivo image quality. Contrast-to-noise ratio. The CNR was calculated in the same way as described above for the mini Derenzo phantoms. However, instead of using the ROIs for one specific rod diameter $d$, the ROIs for the respective animal scan were employed.

Visual image quality assessment. All images were sent to three independent readers for visual assessment of image quality as vertical long axis view (heart) and coronal view (bone). The images were doubled and randomized. Observers were blinded to the acquisition and post-processing protocol and were asked to rate the overall image quality on a five-point scale ( 1 = "very poor", 2 = "poor", 3 = "moderate", 4 = "good", 5 = "very good").

Statistics. Statistical analysis was carried out with specialized software (SPSS Statistics Version 27 for MacOS, IBM, Amonk, New York, USA). Kolmogorov-Smirnov tests were applied to assess normal distribution of continuous variables. Categorical variables are presented as percentages, frequencies and median values with interquartile range (IQR), e.g. for image quality scale results.

\section{Results}

Performance measurements. Point source sensitivity examined with technetium-99m for the UHR-RM collimator was $567 \mathrm{cps} / \mathrm{MBq}(0.057 \%)$. In visual analysis of the hot-rod phantoms' rod sections, a minimum diameter of $1.2 \mathrm{~mm}$ could be discriminated, what was assumed to represent the maximum resolution (Fig. 3a). Uniformity for the UHR-RM collimator was 55.5\% in accordance with the NEMA protocol. Figure 3b,c illustrate reconstructed image and corresponding line profile.

In vitro image quality. Figure 4a provides an illustration of the phantom images used for CNR analysis. For the lower activity an increased image noise is visible, while contrast remains fairly constant.

Figure $4 \mathrm{~b}$ shows the dependency of the $\mathrm{CNR}$ on the investigated rod size for four different activity concentrations. While measurements in rods smaller than $1.2 \mathrm{~mm}$ mostly resulted in very low, partly negative CNR values $(-0.31$ to 0.48$)$, only $300 \mathrm{MBq} / \mathrm{mL}$ achieved a higher value below the maximum resolution $(1.41$ at $1.0 \mathrm{~mm})$. CNR of 1.50 was achieved for $300 \mathrm{MBq} / \mathrm{mL}, 1.0 \mathrm{MBq} / \mathrm{mL}, 0.5 \mathrm{MBq} / \mathrm{mL}$ and $0.1 \mathrm{MBq} / \mathrm{mL}$ at rod sizes of $1.2 \mathrm{~mm}$, $1.8 \mathrm{~mm}, 2.0 \mathrm{~mm}$ and $2.8 \mathrm{~mm}$, respectively. For all activity concentrations, CNR improved continuously with increasing rod size and Gaussian filtering.

In vivo image quality. $\quad\left[{ }^{99 m} T c\right] T c-M I B I$ cardiac rat imaging. SPECT images of the investigated healthy rat heart for different acquisition times $(10,30$ and $60 \mathrm{~min})$ and various post-reconstruction Gaussian filters (FWHM of 1.2, 2.2, 2.8, 3.5 and $4.0 \mathrm{~mm}$ ) are displayed in vertical long axis view in Fig. 5a. The images from all three perspectives (horizontal long axis, short axis, vertical long axis) are shown in supplemental Fig. 1. No artifacts were detected. Left ventricle of the heart is clearly visualized as the myocardium shows sufficient uptake of [ $\left.{ }^{99 \mathrm{~m}} \mathrm{Tc}\right] \mathrm{Tc}-\mathrm{MIBI}$. Images of each scan time without any filtering result in poor image quality and high noise. Gaussian post-filtering clearly improved image quality for each scan.

The results for the in vivo contrast-to-noise ratio and image quality assessment of the healthy rat heart are summarized in Fig. 5b,c. Increasing the FWHM up to $3.5 \mathrm{~mm}$ of the Gaussian filtering results in improved CNR. The highest values were achieved for the 10 -min scan with $4.0 \mathrm{~mm}$ and for the 30 -min and 60 -min scan with $3.5 \mathrm{~mm}$ kernel size, respectively (Fig. 5b). Comparing the three different acquisition times and therefore different count levels, peak values for CNR increased substantially from $10 \mathrm{~min}$ (6.3) to $60 \mathrm{~min}$ (12.2).

Peak image quality scores for the investigated scan times were $5.00(\mathrm{IQR}=0.00)$ for $10 \mathrm{~min}$ and $30 \mathrm{~min}$ with FWHM of 3.5 and $4.0 \mathrm{~mm}, 5.00(\mathrm{IQR}=0.75)$ for $60 \mathrm{~min}$ with FWHM of $4.0 \mathrm{~mm}$ (Fig. 6c). All images without filtering received the lowest rating "very poor". FWHM $=1.2 \mathrm{~mm}$ scored in the range of "very poor" to "moderate", $2.2 \mathrm{~mm}$ "poor" to "good" and $2.8 \mathrm{~mm}$ "moderate" to "very good". 

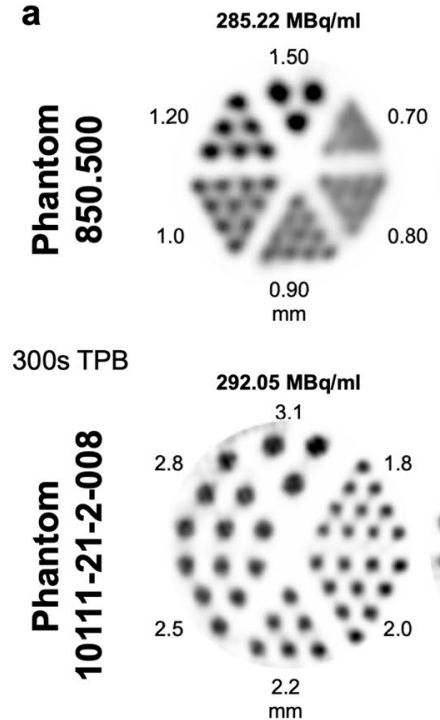
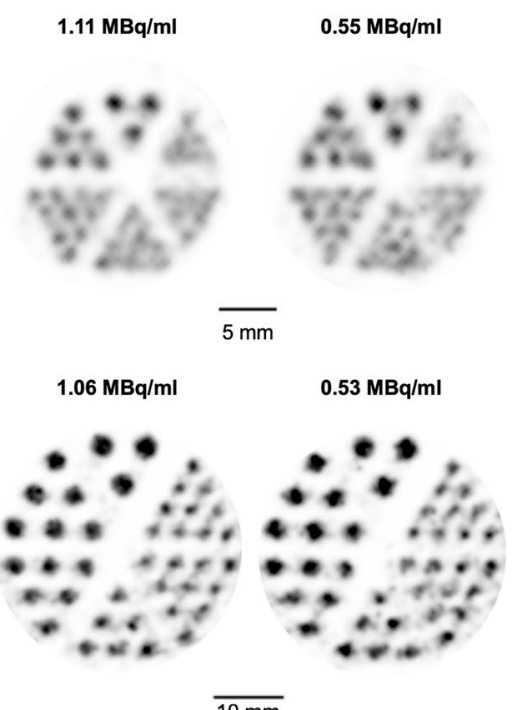

$\overline{10 \mathrm{~mm}}$
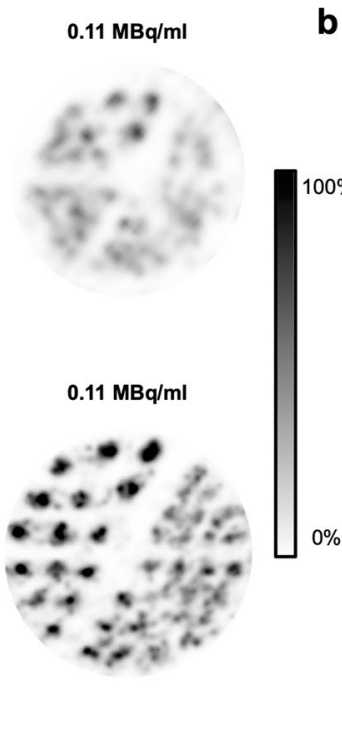
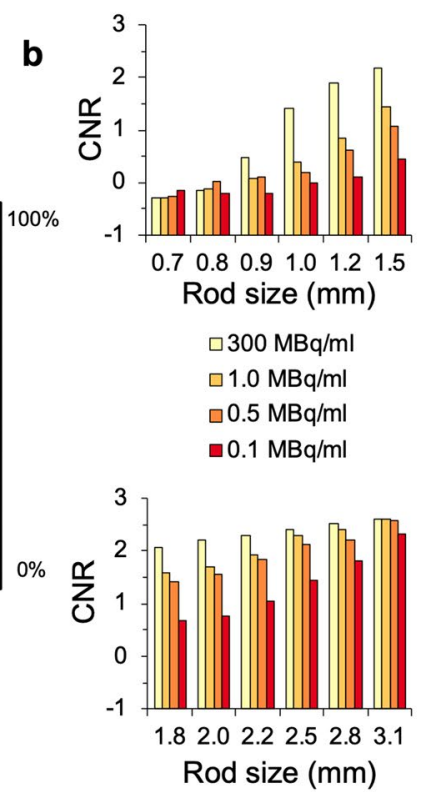

Figure 4. Hot-rod phantom scans and corresponding contrast-to-noise ratios. (a) SPECT images of phantoms 850.500 and 10111-21-2-008 with one high and three low activity ${ }^{99 m} \mathrm{Tc}$-solutions for a $300 \mathrm{~s}$ time per bed position (TPB) scan ( 9 bed positions for the $850.500,6$ bed positions for the 10111-21-2-008). Intensity scale was adapted for each image to enhance contrast. Results are shown in transaxial view with slice thickness of $6.0 \mathrm{~mm}$. Gaussian post-filter of full width at half maximum $(\mathrm{FWHM})=0.7 \mathrm{~mm}$ was applied. (b) Bar charts display corresponding contrast-to-noise ratio of phantom scans optimized for each rod size by Gaussian postfilter of FWHM = rod size.
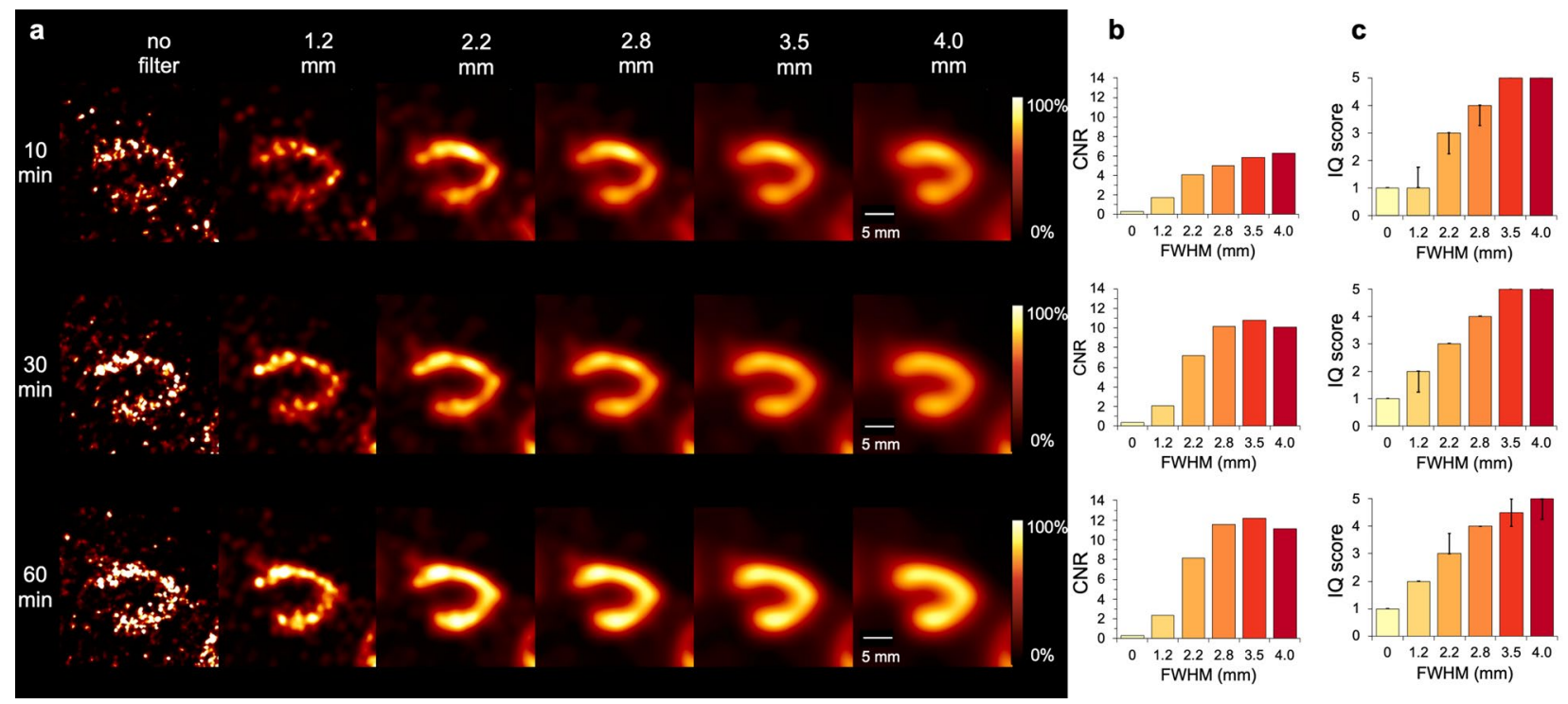

Figure 5. Heart scan assessment. (a) Myocardial perfusion SPECT with three different scan times and six different Gaussian post-filters (FWHM = full width at half maximum). Vertical long axis perspective images have a slice thickness of $0.4 \mathrm{~mm}$ with adjusted color scale. [ ${ }^{99 \mathrm{~m}} \mathrm{Tc}$ ] Tc-MIBI injection dose was $108.5 \mathrm{MBq}$, and the scan was performed $25 \mathrm{~min}$ after tracer injection into tail vein for 60 -min acquisition (40 bed positions, $15 \mathrm{~s}$ time per bed position). Image quality analysis is based on images reconstructed from extracted scan data of $10 \mathrm{~min}, 30 \mathrm{~min}$ and $60 \mathrm{~min}$. (b) Results of corresponding contrast-to-noise ratio calculations. (c) Image quality scores given by three independent readers displayed as median with interquartile range in bar charts.

$\left.{ }^{99 m} \mathrm{Tc}\right] \mathrm{Tc}-\mathrm{HMDP}$ bone rat imaging. Reconstructed images of the healthy rat's lower spine and pelvis region with various Gaussian filters (FWHM =1.2, 1.8, 2.2, 2.8 and $3.5 \mathrm{~mm}$ ) and three different scan times (5, 30 and $90 \mathrm{~min})$ are shown from the coronal view in Fig. 6a. The images from all three perspectives (transverse, coronal, sagittal) are shown in supplemental Fig. 2. No artifacts were detected. Differentiation of vertebra and intervertebral discs was feasible, the pelvic bones were visualized in detail. Images without any filtering appeared noisy and provided 
a

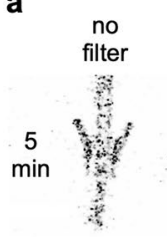

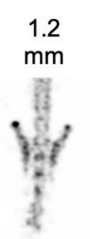
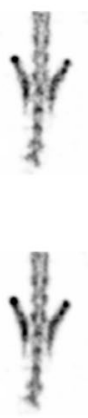
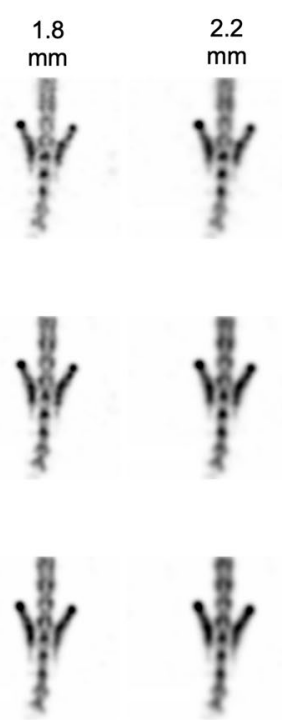

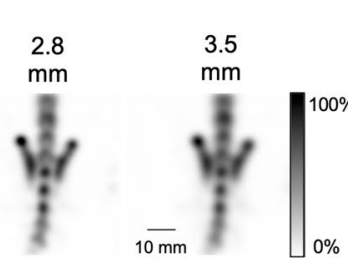

b
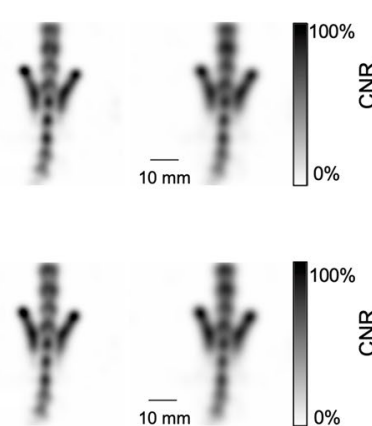
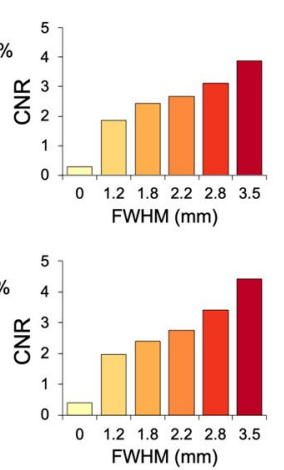

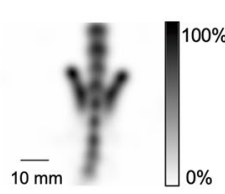

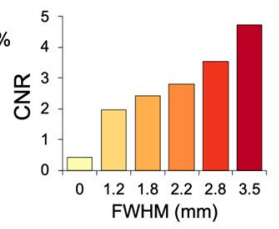
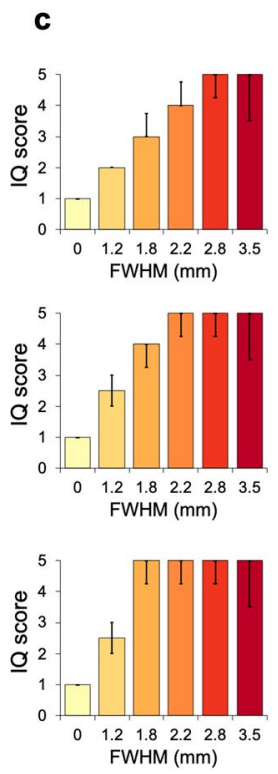

Figure 6. Bone scan assessment. (a) Bone SPECT images with three different scan times and six different Gaussian post-filters (FWHM = full width at half maximum). The scan was focused on pelvis and lower spine of a healthy rat, the top-view perspective is shown with a slice thickness of $0.4 \mathrm{~mm}$ and adjusted scaling for good contrast. [ ${ }^{99 \mathrm{~m}} \mathrm{Tc}$ ] Tc-HMDP injection dose was $154.9 \mathrm{MBq}$, and the scan was performed 60 min after tracer injection into tail vein for $90 \mathrm{~min}$ acquisition (15 bed positions, $20 \mathrm{~s}$ time per bed position). Image quality analysis is based on images reconstructed from extracted scan data of $5 \mathrm{~min}, 30 \mathrm{~min}$ and $90 \mathrm{~min}$. (b) Results of corresponding contrast-to-noise ratio calculations. (c) Image quality scores given by three independent readers displayed as median with interquartile range in bar charts.

poor detail. Nevertheless, higher count levels and longer acquisition time led to an overall improvement of image quality. By increasing the post-reconstruction Gaussian filter, image quality was further enhanced. High filtering resulted in homogenous and smooth images but provided less detail and seemed overly blurred.

Figure $6 \mathrm{~b}$ depicts results of the in vivo CNR analysis. Irrespective of acquisition time, Gaussian filtering of $3.5 \mathrm{~mm}$ was associated with the highest CNR values. However, no clear difference in absolute CNR values could be determined by extending the acquisition time.

Figure $6 \mathrm{c}$ plots the image quality scores of images shown in Fig. $6 \mathrm{a}$ as a function of Gaussian kernel size. Peak image quality scores of 5.00 for the investigated scan times were achieved for 5 min and FWHM of 2.8 and $3.5 \mathrm{~mm}$, for $30 \mathrm{~min}$ and FWHM of 2.2, 2.8 and $3.5 \mathrm{~mm}$ and for $90 \mathrm{~min}$ and FWHM $\geq 1.8 \mathrm{~mm}$. Quality of unfiltered images scored $1.00(\mathrm{IQR}=0.00)$ for every acquisition time by all readers. Ratings for kernel size $1.2 \mathrm{~mm}$ ranged from "poor" to "moderate". Image quality for FWHM of $1.8 \mathrm{~mm}$ was considered "moderate" to "good". The images filtered with a kernel size of 2.2, 2.8 and $3.5 \mathrm{~mm}$ were all rated "moderate" to "very good".

\section{Discussion}

We investigated to what extent the recently introduced two-detector U-SPECT5-E with UHR-RM collimator and the novel SROSEM algorithm is suitable for ultra-high-resolution rat imaging in a realistic in vivo setting.

In the performance evaluation, the SPECT system achieved a sensitivity of $567 \mathrm{cps} / \mathrm{MBq}$, a resolution of $1.2 \mathrm{~mm}$ and a uniformity of $55.5 \%$. This was comparable to the previous generation with three detectors and a similar multi-pinhole rat collimator, which achieved a sensitivity of $700 \mathrm{cps} / \mathrm{MBq}$ and a resolution of $0.8 \mathrm{~mm}$. It should be noted that an activity concentration of $600 \mathrm{MBq} / \mathrm{mL}$ was used ${ }^{10}$. No direct comparison was made between the two-detector system and the three-detector system regarding the rat imaging capabilities. Such comparison might reveal the impact of the missing lower detector in more detail. Boisson et al. evaluated a system with rotating detectors and three different rat collimators with maximum resolutions in the range of 1.1-2.0 mm but sensitivities below $300 \mathrm{cps} / \mathrm{MBq}$. They only used either one or three pinholes ${ }^{28}$. For another established system with four detectors, three rat collimators were reported with maximum resolutions in the range of 1.1-1.9 $\mathrm{mm}$ and sensitivities of up to more than $2000 \mathrm{cps} / \mathrm{MBq}^{29}$, but more detailed methodological information is lacking and the recently published study on the new generation of the system contains mainly information on the mouse collimators ${ }^{30}$. However, it should be noted that the activity concentrations in these performance evaluations using phantoms are much higher than the activity concentrations under realistic in vivo conditions.

In the in vitro CNR analysis, values were considerably lower for small rod sizes and lower activity concentrations and increased with larger rod size and post-filtering. Similar observations were made for pinhole PET by Walker et al. ${ }^{25}$. The Gaussian post-filtering results in a maximum resolution (FWHM) limited by the kernel size of the filter. The CNR values in phantom measurements (Fig. 4) were substantially lower at low activities compared to high activities. To achieve a CNR similar to values in rods of $1.2 \mathrm{~mm}$ at $300 \mathrm{MBq} / \mathrm{mL}$, larger rods and filters 
were required in the in vivo study count range. Hence, reasonable CNR values were achieved for $1.0 \mathrm{MBq} / \mathrm{mL}$ with $\geq 1.8 \mathrm{~mm}$, for $0.5 \mathrm{MBq} / \mathrm{mL}$ with $\geq 2.0 \mathrm{~mm}$ and for $0.1 \mathrm{MBq} / \mathrm{mL}$ with $\geq 2.8 \mathrm{~mm}$ rod size.

Multi-observer analysis of the longest in vivo scans (60 and $90 \mathrm{~min})$ gives a count range comparable to approximately $1.0 \mathrm{MBq} / \mathrm{mL}$ in phantom studies. Good CNR and image quality scores were found for the SPECT images with Gaussian kernels bigger than $1.8 \mathrm{~mm}$ in bone and $2.8 \mathrm{~mm}$ in the rat heart. This implies that the maximum resolution that can realistically be achieved for in vivo imaging is slightly lower than the system resolution of $1.2 \mathrm{~mm}$.

Analysis of in vivo heart studies revealed a substantial increase in CNR between 10 and 30 min, while extension of acquisition time to $60 \mathrm{~min}$ resulted in only a minor increase. A similar tendency was seen in the visual image analysis, where the scan time did not lead to a relevant image quality increase and the longest scan time of 60 min even resulted in inferior image quality ratings. This loss of image quality could be the result of slightly more intensive scaling of the $60 \mathrm{~min}$ images. In contrast to acquisition time, the choice of filter size had considerably more effect on subjective ratings. While $4.0 \mathrm{~mm}$ led to the best results for short scan times in the heart, the best CNR value could be achieved with a longer scan time and a filter of $3.5 \mathrm{~mm}$. The filters of $3.5 \mathrm{~mm}$ or $4.0 \mathrm{~mm}$ also provided the best subjective image quality for professional readers. Nonetheless, even a filter of $2.2 \mathrm{~mm}$ delivered acceptable image quality and can be suitable if the additional resolution is required for certain imaging tasks. Mizutani et al. also investigated the influence of injection dose and post-filtering on image quality in the rat heart. A SPECT system with cadmium-zinc telluride detectors and two different five-pinhole collimators was used (sensitivity $=321 \mathrm{cps} / \mathrm{MBq}, 139 \mathrm{cps} / \mathrm{MBq}$; resolution $=1.5 \mathrm{~mm}, 1.2 \mathrm{~mm}$ ). The evaluation by two readers showed an increase in image quality by higher injection dose $(25-200 \mathrm{MBq})$ and stronger filters (no filter; FWHM $=1.5 \mathrm{~mm}, 2.5 \mathrm{~mm})^{14}$. The injection dose had more impact on the image quality score than we found for the scan time, but the results are still largely consistent with ours.

In the case of bone imaging, stronger filtering increased CNR without restriction, although the anatomical structures appeared blurry throughout. The bone structure in the area of the lumbar spine and pelvis is very precise and complex, which might lead to a supposedly high noise level in the calculation. On the other hand, observer analysis showed no considerable increase between the different scan times of 5, 30 and 90 min, while increasing the Gaussian filter from $1.2 \mathrm{~mm}$ to $1.8 \mathrm{~mm}$ leads to a noticeably higher image quality score. The positive effect of post-filtering on CNR and image quality can most likely be attributed to the improvements in uniformity and noise ${ }^{13}$. The study is limited by the use of only one rat per tracer. A certain inter-animal variance could be expected, since we focused on the impact of scan time and post filter on the image quality and not the quantitative capabilities, we decided to use only two examples with well-established tracers for reasons of good animal practice.

\section{Conclusion}

We analyzed the performance of a recently introduced ultra-high-resolution micro-SPECT system with two stationary detectors for preclinical rat imaging. Providing good image quality with a multi-pinhole UHR-RM collimator, the scanner is suitable for heart and bone scans using standardized reconstruction parameters and appropriate post-filtering. Although the system demonstrates excellent performance in rat imaging as compared to conventional systems, there are still challenges to achieve sub-millimeter system resolution in rats where there are safety limits on injection dose and acquisition time.

\section{Data availability}

The datasets generated and/or analyzed during the current study are available from the corresponding author on reasonable request.

Received: 27 July 2020; Accepted: 7 October 2020

Published online: 29 October 2020

\section{References}

1. King, M. A., Pretorius, P. H., Farncombe, T. \& Beekman, F. J. Introduction to the physics of molecular imaging with radioactive tracers in small animals. J. Cell Biochem. Suppl. 39, 221-230. https://doi.org/10.1002/jcb.10447 (2002).

2. Meikle, S. R., Kench, P., Kassiou, M. \& Banati, R. B. Small animal SPECT and its place in the matrix of molecular imaging technologies. Phys. Med. Biol. 50, R45-61. https://doi.org/10.1088/0031-9155/50/22/R01 (2005).

3. Beekman, F. \& van der Have, F. The pinhole: Gateway to ultra-high-resolution three-dimensional radionuclide imaging. Eur. J. Nucl. Med. Mol. Imaging 34, 151-161. https://doi.org/10.1007/s00259-006-0248-6 (2007).

4. Magota, K. et al. Performance characterization of the Inveon preclinical small-animal PET/SPECT/CT system for multimodality imaging. Eur. J. Nucl. Med. Mol. Imaging 38, 742-752. https://doi.org/10.1007/s00259-010-1683-y (2011).

5. Matsunari, I. et al. Performance evaluation of the eXplore speCZT preclinical imaging system. Ann. Nucl. Med. 28, 484-497. https ://doi.org/10.1007/s12149-014-0828-7 (2014).

6. Ivashchenko, O., van der Have, F., Goorden, M. C., Ramakers, R. M. \& Beekman, F. J. Ultra-high-sensitivity submillimeter mouse SPECT. J. Nucl. Med. 56, 470-475. https://doi.org/10.2967/jnumed.114.147140 (2015).

7. Franc, B. L., Acton, P. D., Mari, C. \& Hasegawa, B. H. Small-animal SPECT and SPECT/CT: Important tools for preclinical investigation. J. Nucl. Med. 49, 1651-1663. https://doi.org/10.2967/jnumed.108.055442 (2008).

8. Furenlid, L. R. et al. FastSPECT II: A second-generation high-resolution dynamic SPECT imager. IEEE Trans. Nucl. Sci. 51, 631-635. https://doi.org/10.1109/TNS.2004.830975 (2004).

9. Vastenhouw, B. \& Beekman, F. Submillimeter total-body murine imaging with U-SPECT-I. J. Nucl. Med. 48, 487-493 (2007).

10. van der Have, F. et al. U-SPECT-II: An ultra-high-resolution device for molecular small-animal imaging. J. Nucl. Med. 50, 599-605. https://doi.org/10.2967/jnumed.108.056606 (2009).

11. Ivashchenko, O. et al. Quarter-millimeter-resolution molecular mouse imaging with U-SPECT(+). Mol.S Imaging 13, 10. https:// doi.org/10.2310/7290.2014.00053 (2014). 
12. Higaki, Y. et al. Appropriate collimators in a small animal SPECT scanner with CZT detector. Ann. Nucl. Med. 27, 271-278. https ://doi.org/10.1007/s12149-012-0681-5 (2013).

13. Harteveld, A. A. et al. Using the NEMA NU 4 PET image quality phantom in multipinhole small-animal SPECT. J. Nucl. Med. 52, 1646-1653. https://doi.org/10.2967/jnumed.110.087114 (2011).

14. Mizutani, A. et al. Impact of injection dose, post-reconstruction filtering, and collimator choice on image quality of myocardial perfusion SPECT using cadmium-zinc telluride detectors in the rat. EJNMMI Phys. 2, 7. https://doi.org/10.1186/s40658-015-0111-6 (2015).

15. Vaissier, P. E., Beekman, F. J. \& Goorden, M. C. Similarity-regulation of OS-EM for accelerated SPECT reconstruction. Phys. Med. Biol. 61, 4300-4315. https://doi.org/10.1088/0031-9155/61/11/4300 (2016).

16. Iannaccone, P. M. \& Jacob, H. J. Rats!. Dis. Model Mech. 2, 206-210. https://doi.org/10.1242/dmm.002733 (2009).

17. Jones, A. G. et al. Biological studies of a new class of technetium complexes: The hexakis(alkylisonitrile)technetium(I) cations. Int. J. Nucl. Med. Biol. 11, 225-234. https://doi.org/10.1016/0047-0740(84)90004-4 (1984).

18. Bevan, J. A., Tofe, A. J., Benedict, J. J., Francis, M. D. \& Barnett, B. L. Tc-99m HMDP (hydroxymethylene diphosphonate): A radiopharmaceutical for skeletal and acute myocardial infarct imaging. I. Synthesis and distribution in animals. J. Nucl. Med. 21, 961-966 (1980).

19. Bevan, J. A., Tofe, A. J., Benedict, J. J., Francis, M. D. \& Barnett, B. L. Tc-99m HMDP (hydroxymethylene diphosphonate): A radiopharmaceutical for skeletal and acute myocardial infarct imaging. II. Comparison of Tc-99m hydroxymethylene diphosphonate (HMDP) with other technetium-labeled bone-imaging agents in a canine model. J. Nucl. Med. 21, 967-970 (1980).

20. Vaissier, P. E. et al. Fast spiral SPECT with stationary gamma-cameras and focusing pinholes. J. Nucl. Med. 53, 1292-1299. https ://doi.org/10.2967/jnumed.111.101899 (2012).

21. Shepp, L. A. \& Vardi, Y. Maximum likelihood reconstruction for emission tomography. IEEE Trans Med. Imaging 1, 113-122. https ://doi.org/10.1109/TMI.1982.4307558 (1982).

22. Ogawa, K., Harata, Y., Ichihara, T., Kubo, A. \& Hashimoto, S. A practical method for position-dependent Compton-Scatter correction in single photon emission CT. IEEE Trans. Med. Imaging 10, 408-412. https://doi.org/10.1109/42.97591 (1991).

23. Loening, A. M. \& Gambhir, S. S. AMIDE: A free software tool for multimodality medical image analysis. Mol. Imaging 2, 131-137. https://doi.org/10.1162/153535003322556877 (2003).

24. National Electrical Manufacturers Association in NEMA Standards Publication NU 4-2008: Performance Measurements of Small Animal Positron Emission Tomographs (Rosslyn, VA, 2008).

25. Walker, M. D. et al. Performance assessment of a preclinical PET scanner with pinhole collimation by comparison to a coincidencebased small-animal PET scanner. J. Nucl. Med. 55, 1368-1374. https://doi.org/10.2967/jnumed.113.136663 (2014).

26. Bayne, K. Revised guide for the care and use of laboratory animals available. American Physiological Society. Physiologist 39(199), 208-111 (1996).

27. Arsos, G. et al. (99m)Tc-sestamibi uptake in rat skeletal muscle and heart: Physiological determinants and correlations. Physiol. Res. 58, 21-28 (2009).

28. Boisson, F. et al. Imaging capabilities of the Inveon SPECT system using single-and multipinhole collimators. J. Nucl. Med. 54, 1833-1840. https://doi.org/10.2967/jnumed.112.117572 (2013).

29. Schramm, N. et al. Improving resolution, sensitivity and applications for the NanoSPECT/CT: A high-performance SPECT/CT imager for small-animal research. J. Nucl. Med. 48, 436P (2007).

30. Lukas, M., Kluge, A., Beindorff, N. \& Brenner, W. Multi-isotope capabilities of a small-animal multi-pinhole SPECT system. J. Nucl. Med. 61, 152-161. https://doi.org/10.2967/jnumed.119.226027 (2020).

\section{Acknowledgements}

We would like to thank the Department of Nuclear Medicine of the University Hospital Würzburg for providing the tracers. Jan Vincent Hoffmann and Jan Paul Janssen were supported by a doctoral fellowship of the Faculty of Medicine, University of Würzburg in the framework of the Graduate School of Life Sciences. Takayuki Kanno was supported by the Japan Public-Private Partnership Student Study Abroad Program (TOBITATE! Young Ambassador Program). The authors of this manuscript declare no relationships with any companies, whose products or services may be related to the subject matter of the article.

\section{Author contributions}

Conception: J.P.J., J.V.H., T.H.; Acquisition, analysis and interpretation: J.P.J., J.V.H., T.K.; Writing—original draft preparation: J.P.J., J.V.H.; Writing-review and editing: J.P.J., J.V.H., T.K., N.N., J.G., M.O., X.C., C.L., A.B., T.H.; Funding acquisition: J.P.J., J.V.H., T.K., C. L., T.H., A.B.; Supervision: T.H., A.B.; Validation: N.N., J.G., M.O., X.C., C.L., A.B., T.H.; Visualization: J.P.J., J.V.H.

\section{Funding}

Open Access funding enabled and organized by Projekt DEAL.

\section{Competing interests}

The authors declare no competing interests.

\section{Additional information}

Supplementary information is available for this paper at https://doi.org/10.1038/s41598-020-75696-0.

Correspondence and requests for materials should be addressed to T.H.

Reprints and permissions information is available at www.nature.com/reprints.

Publisher's note Springer Nature remains neutral with regard to jurisdictional claims in published maps and institutional affiliations. 
(c) (i) Open Access This article is licensed under a Creative Commons Attribution 4.0 International cc) License, which permits use, sharing, adaptation, distribution and reproduction in any medium or format, as long as you give appropriate credit to the original author(s) and the source, provide a link to the Creative Commons licence, and indicate if changes were made. The images or other third party material in this article are included in the article's Creative Commons licence, unless indicated otherwise in a credit line to the material. If material is not included in the article's Creative Commons licence and your intended use is not permitted by statutory regulation or exceeds the permitted use, you will need to obtain permission directly from the copyright holder. To view a copy of this licence, visit http://creativecommons.org/licenses/by/4.0/.

(C) The Author(s) 2020 